\title{
Preparation of Thick Silica Films by the Electrophoretic Sol-Gel Deposition Using a Cationic Polymer Surfactant
}

\author{
Koichi HASEGAWA, Masahiro TATSUMISAGO and Tsutomu MINAMI \\ Department of Applied Materials Science, College of Engineering, Osaka Prefecture University, 1-1, Gakuen-cho, Sakai-shi, Osaka \\ 593
}

\author{
カチオン系高分子界面活性剤を用いたゾルーゲル電気泳動電着によるシリカ厚膜の作製 \\ 長谷川公一・辰巳砂昌弘・南＼cjkstart努 \\ 大阪府立大学工学部機能物質科学科, 593 大阪府堺市学園町 1-1
}

\begin{abstract}
Thick silica films were prepared on the cathode by the electrophoretic sol-gel deposition using a cationic polymer surfactant, polyethylenimine (PEI). The weight of the silica particles deposited on the cathode was maximized when the content of PEI added was 0.01 mass $\%$ and $\mathrm{pH}$ of the coating sols was 5 . As the amount of PEI added increased over the optimum content $(0.01$ mass $\%)$, the weight of the silica particles deposited decreased with an increase in PEI content because of $\mathrm{H}_{2}$ generation at the cathode and the aggregation and precipitation of silica particles in the coating sols. Thick silica films obtained had no cracks and the thickness was ca. $20 \mu \mathrm{m}$.

[Received January 27, 1997; Accepted April 1, 1997]
\end{abstract}

Key-words : Electrophoretic sol-gel deposition, Silica, Thick film, Polyethylenimine, Polymer surfactant

\section{Introduction}

Electrophoretic sol-gel deposition is one of the useful methods to prepare thick silica films. ${ }^{1)}$ Silica films with more than $10 \mu \mathrm{m}$ in thickness have been obtained by the electrophoretic deposition, whereas it is difficult to obtain such thick films by dip-coating or spin-coating.

Since the isoelectric point of silica particles is about $\mathrm{pH}$ $2-3$ in water, the particles in sols have large negative charge at neutral and basic conditions. As a result, silica particles are usually deposited on an anode by applying d.c. voltage. However, an anodic deposition has been pointed out to have some critical disadvantages: elution of metal ions of the anode material into coating sols, incorporation of the eluted metal ions into films obtained, etc. Such a contamination in the coating films sometimes causes the lowering of electric insulation and the coloration of films. ${ }^{2), 3)}$ In contrast, a cathodic deposition has widely been accepted especially in industry because these disadvantages can be avoided. Since the isoelectric point of silica particles is about $\mathrm{pH}$ $2-3$, strong acidic condition can yield positively charged silica particles. However, the positive charge of silica particles is not so large in such a case and the electrodes are easily corroded by the strong acidic sols. Thus, it is difficult to obtain positively charged silica particles only by controlling $\mathrm{pH}$ of the coating sols.

The objective of this study is to prepare thick silica films on cathodes by electrophoretic sol-gel deposition. We used a cationic polymer surfactant, polyethylenimine (PEI), in the electrophoretic sol-gel deposition in order to control the surface charge of silica particles. The positive charge of PEI can be controlled by altering $\mathrm{pH}$. Furthermore, the amount of PEI adsorbed on negatively charged silica particles may be changed with $\mathrm{pH}$ of these sols. ${ }^{4)}$ Consequently, the surface charge of silica particles can be reversed from negative to positive by adding a suitable amount of PEI at an appropriate value of $\mathrm{pH}$.

This paper reports the effects of $\mathrm{pH}$ of the coating sols and the amount of PEI added on the weight of the silica particles deposited on the cathode in the electrophoretic solgel deposition. The particle dispersibility and the particle arrangement at the surface of the thick silica films obtained are also discussed.

\section{Experimental}

Silica particles used in this study were prepared by the procedure which we previously reported. ${ }^{5), 6)}$ Silica particles obtained were monodispersed with the averaged diameter of $170 \mathrm{~nm}$. A mixture of $\mathrm{H}_{2} \mathrm{O}$ and $\mathrm{C}_{2} \mathrm{H}_{5} \mathrm{OH}$, the molar ratio of which was $1 / 4$, was used as a dispersion medium of the particles. The amount of silica particles added was fixed to be 1 mass $\%$ and that of PEI ranged from 0 to 1.00 mass $\%$ in the medium. The total weight of the mixture was $70 \mathrm{~g}$. The mixture was well stirred with a magnetic stirrer and ultrasonication. Dilute nitric acid was used to control $\mathrm{pH}$ of the mixture. Silica suspension obtained was used as a coating sol for electrophoretic deposition. A stainless steel sheet (SUS430) was used as a coating electrode and a stainless steel wire (SUS304BA) was used as a counter electrode. Electrophoretic deposition was carried out by applying 40 $\mathrm{V}$. The weight of the silica films deposited was measured by a micro-balance (Mettler M5SA). The diameter and zeta potentials of the silica particles were measured by using an instrument for particle size and for zeta potential measurements (Malverm Instrument Ltd., Zetamaster). The dynamic light scattering system was adopted for the measurement of the particle size in the coating sols and the laser doppler system was used for the measurement of zeta potentials of the particles. Observation of the microstructure for the surface and the cross section of the thick silica films was performed by a scanning electron microscope (SEM; JEOL, JSM-5300). The thickness of the films was also estimated by the SEM observation of the cross section.

\section{Results and discussion}

Figure 1 shows the weight of the silica particles deposited on an anode or a cathode as a function of deposition time for the amount of PEI added of 0 (open circles) and of 0.01 (closed circles) mass\%. The weight of the silica particles deposited on the anode is denoted in the right axis and that on the cathode in the left axis. No silica particles were deposited on the cathode unless PEI was added and no particles were deposited on the anode when 0.01 mass \% PEI was added. The value of $\mathrm{pH}$ of the dispersion medium was adjusted to be 6 . Without PEI, small amounts of silica particles were deposited on the anode. However, large amounts of silica particles were deposited on the cathode when 0.01 


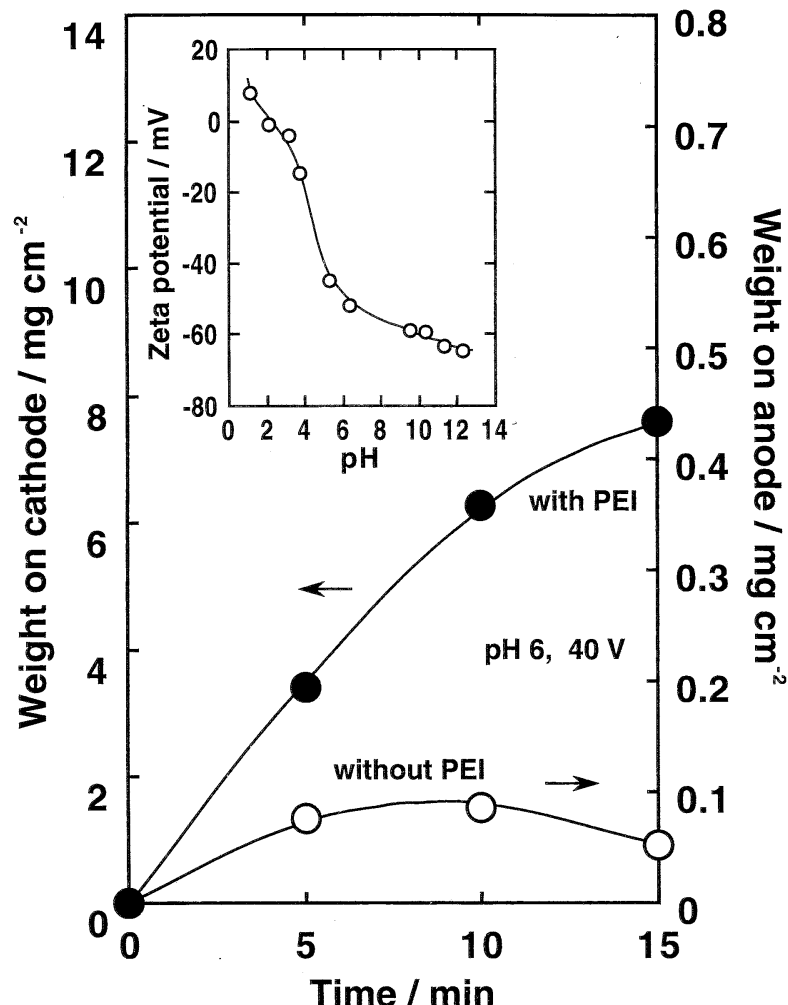

Fig. 1. Weight of the silica particles deposited on the cathode (closed circle) with the addition of 0.01 mass\% PEI, and on the anode (open circle) without PEI. Insert is the zeta potentials of the silica particles dispersed in water.

mass \% PEI was added, indicating that the surface charge of silica particles was inversed from negative to positive by the addition of cationic surfactant PEI. The insert shows the zeta potentials against $\mathrm{pH}$ of dispersion water for the silica particles used. Since the isoelectric point of the silica particles is about $\mathrm{pH} 2.5$, the negative charge of the particles in the dispersing water is relatively large at $\mathrm{pH} 6$. Without PEI, negatively charged silica particles were deposited on the anode at $\mathrm{pH} 6$, as shown by the open circles in Fig. 1. Since PEI in the coating sols is protonated and therefore positively charged at $\mathrm{pH} 6, \mathrm{PEI}$ is considered to be strongly adsorbed on silica particles by electrostatic attraction. As a result, silica particles migrated toward the cathode and brought about a large amount of deposition, as shown by the closed circles in Fig. 1.

Figure 2 shows the weight of the silica particles deposited on the cathode as a function of $\mathrm{pH}$ of the coating sols. The weight of the silica particles deposited is maximized at $\mathrm{pH}$ 5. The decrease in weight of the particles at $\mathrm{pH}<5$ is due to the smaller negative charge of the silica particles as $\mathrm{pH}$ of the coating sols becomes smaller, as shown in the insert of Fig. 1. As a result, it becomes difficult for the positively charged PEI to be adsorbed on the particles. On the other hand, the weight is gradually decreased at $\mathrm{pH}>5$. Since the degree of protonation of PEI is reported to be zero at $\mathrm{pH} 10.8,{ }^{4)}$ these decrease is due to the smaller extent of protonation of PEI as $\mathrm{pH}$ of the coating sols becomes larger. At $\mathrm{pH} 5$, thus, the protonation and adsorption of $\mathrm{PEI}$ are both good for obtaining large amounts of silica particles deposited on the cathode.

Figure 3 shows the weight of the silica particles deposited on the cathode as a function of PEI content. The $\mathrm{pH}$ of the coating sols was fixed to be 6 . No deposition of silica particles on the cathode was observed in the absence of PEI.

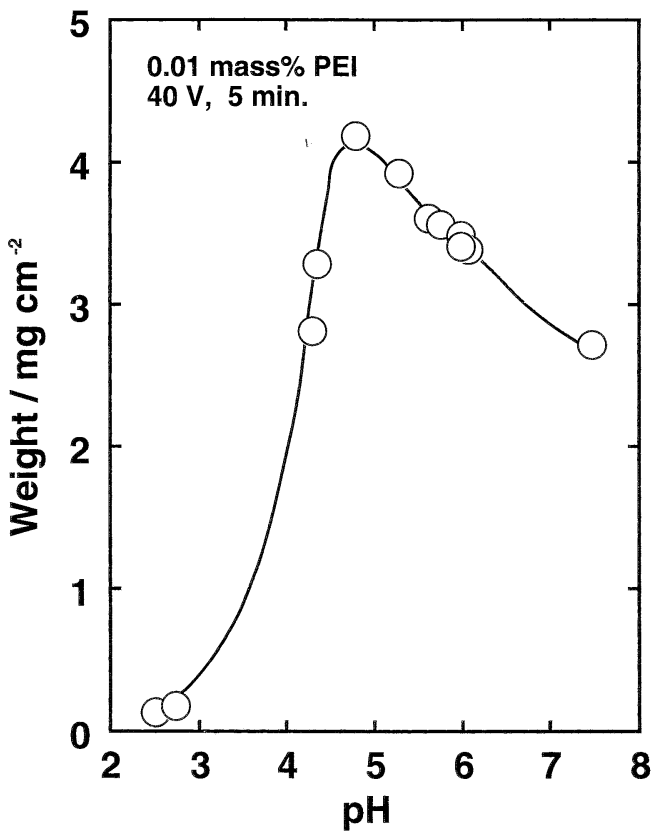

Fig. 2. Weight of the silica particles deposited on the cathode as a function of $\mathrm{pH}$ of the coating sols. Deposition time was $5 \mathrm{~min}$.

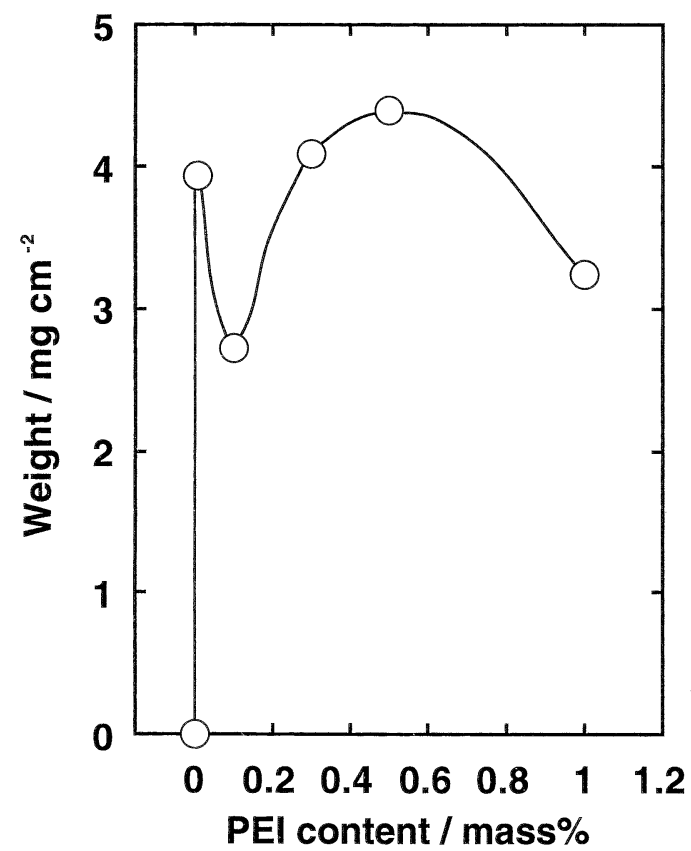

Fig. 3. Weight of the silica particles deposited on the cathode as a function of PEI content. Deposition time was $5 \mathrm{~min}$.

The weight of the silica particles deposited on the cathode increases drastically with the addition of 0.01 mass \% PEI. Further addition of PEI brought about a steep decrease of weight at 0.10 mass \% PEI added. The weight is then gradually increased again by adding 0.50 mass \% PEI. Finally, the weight of the silica particles deposited on the cathode decreases again with 1.00 mass\% PEI. Precipitation of silica particles was observed at the bottom of dispersion vessel for the sols containing 1.00 mass\% PEI. The first drastic increase of weight must be caused by the adsorption of cationic PEI on silica particles and the decrease at the last stage must be caused by the precipitation of the particles in the coating sols. However, much more detailed explanations 


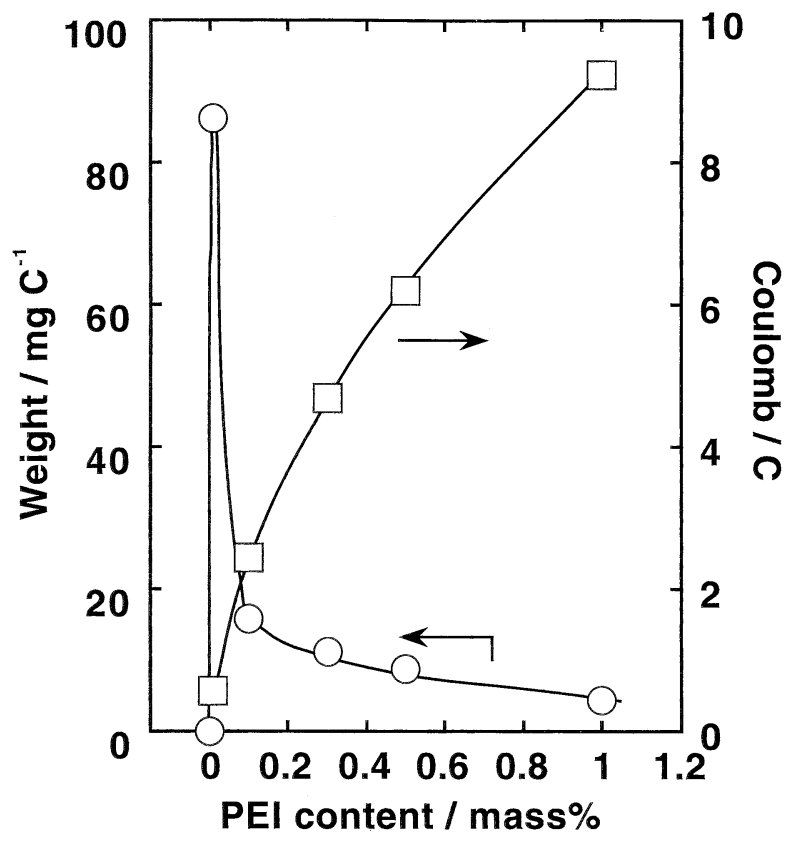

Fig. 4. Weight of the silica particles deposited on the cathode, which is normalized by the electricity supplied (open circle), and total amount of electricity (open square) as a function of PEI content.

are needed to understand these complex variations of the weight of the silica particles deposited on the cathode and will be discussed in the next paragraph together with the results of Fig. 4.

Figure 4 shows the weight of the silica particles deposited on the cathode per coulomb, which is obtained by dividing the total weight deposited by the total electricity supplied. The electricity supplied on 5 min deposition is also shown by open squares. The coulombic efficiency is maximized when the amount of PEI added is 0.01 mass\%. This is consistent with the result that the weight of the silica particles deposited on the cathode is maximized at 0.01 mass $\%$ PEI as shown in Fig. 3. More than 0.01 mass\% PEI results in the monotonous decrease in the coulombic efficiency, which is caused by the generation of $\mathrm{H}_{2}$ gas at the surface of the cathode. Particularly, the decrease of coulombic efficiency is quite steep from 0.01 to 0.10 mass\% PEI, which caused the steep decrease of the weight in the composition region from 0.01 to 0.10 mass \% in Fig. 3. When more than 0.10 mass $\%$ PEI is added, no more decrease of the weight was observed in Fig. 3, which is due to the slight decrease of coulombic efficiency in Fig. 4. An increase of total electricity due to the increase of PEI as a supporting electrolyte leads to the increase of the weight deposited on cathode from 0.10 to 0.50 mass \% PEI in Fig. 3.

Figure 5 shows the size distribution of the silica particles in the coating sols. When the amount of PEI added is 0.01 , 0.10 and 0.50 mass $\%$, the distribution of diameter is relatively sharp and the averaged diameter of the particles is about $170 \mathrm{~nm}$. However, when PEI added is 1.00 mass \%, a broader peak is observed with averaged diameter of 260 $\mathrm{nm}$. Thus silica particles are well dispersed in the case of PEI added less than 0.50 mass $\%$ because the silica particles used in this study was originally $170 \mathrm{~nm}$ in diameter as described in the experimental section. In contrast, silica particles are aggregated in the sols with 1.00 mass \% PEI. This result agrees with the fact that silica particles were precipitated and the weight decreased when PEI added was 1.00 mass $\%$ as shown in Fig. 3.

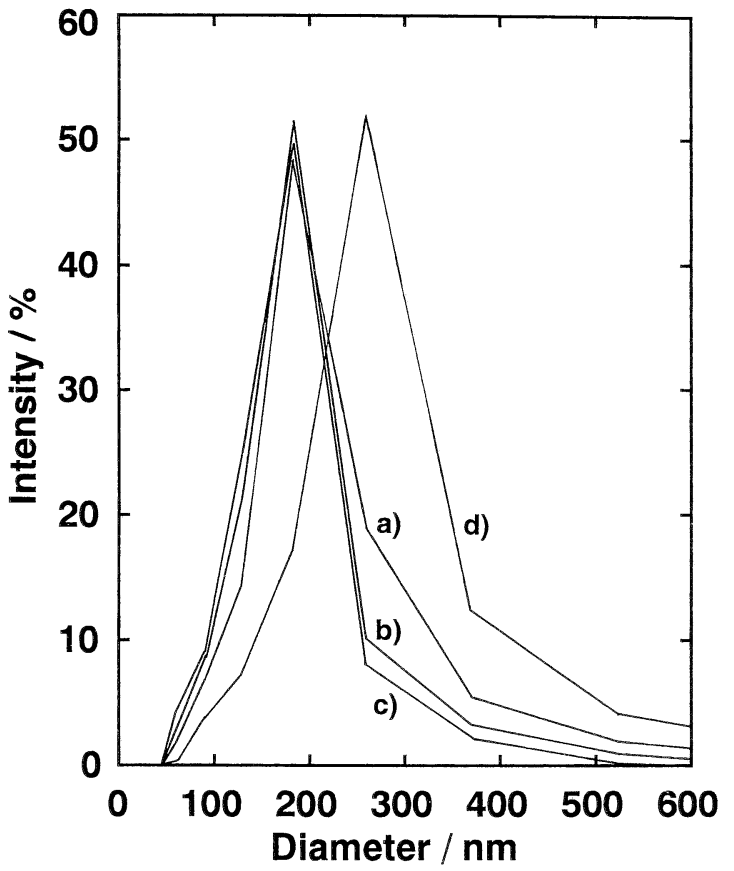

Fig. 5. Size distribution of the silica particles in the coating sols. PEI added was (a) 0.01 , (b) 0.10 , (c) 0.50 and (d) 1.00 mass $\%$.

Figure 6 shows the SEM photographs of the surface of the thick silica films deposited on a stainless steel sheet. No cracks are observed in each film. Silica particles are regularly arranged in the films prepared by adding 0.01 mass $\%$ PEI. When the amount of PEI added increases, particle arrangement becomes irregular. As explained in Fig. $5, \mathrm{H}_{2}$ gas generation at the surface of the cathode became vigorous as the amount of PEI increased. This may cause the irregularity of the particle arrangement. When the content of PEI is 1.00 mass\%, no particles are observed in some regions near the aggregated particles. Only PEI is probably deposited in such regions with no particles. From these photographs, the optimum amount of PEI added is concluded to be 0.01 mass $\%$ to obtain smooth and densely packed silica films.

Figure 7 shows the SEM photograph of the cross section of a thick silica film prepared by adding 0.01 mass \% PEI. The silica film is well adhered on the stainless steel sheet and thickness of the film is ca. $20 \mu \mathrm{m}$.

When the PEI content was 0.01 mass $\%$, the silica particles were regularly arranged on the surface of the films, as shown in Fig. 6. Moreover, the weight of the particles deposited was maximized at this PEI content as shown in Fig. 3. Consequently, optimum amount of PEI added was concluded to be 0.01 mass $\%$ and thick silica films were obtained at cathode as shown in Fig. 7.

\section{Conclusions}

Thick silica films were prepared on a cathode using the electrophoretic sol-gel deposition by adding a cationic polymer surfactant, PEI, into the dispersion medium of silica particles. The weight of the silica particles deposited on the cathode was maximized when $\mathrm{pH}$ of the coating sols was 5 . Silica particles had large positive charge due to both adsorption and protonation of PEI on silica particles at $\mathrm{pH}$ 5. The weight of the silica particles deposited on the cathode was maximized at 0.01 mass \% PEI. As the amount of PEI was increased more than 0.01 mass $\%$, the weight was decreased because of vigorous $\mathrm{H}_{2}$ gas generation at the cathode. The gradual increase of the weight from 0.10 to 

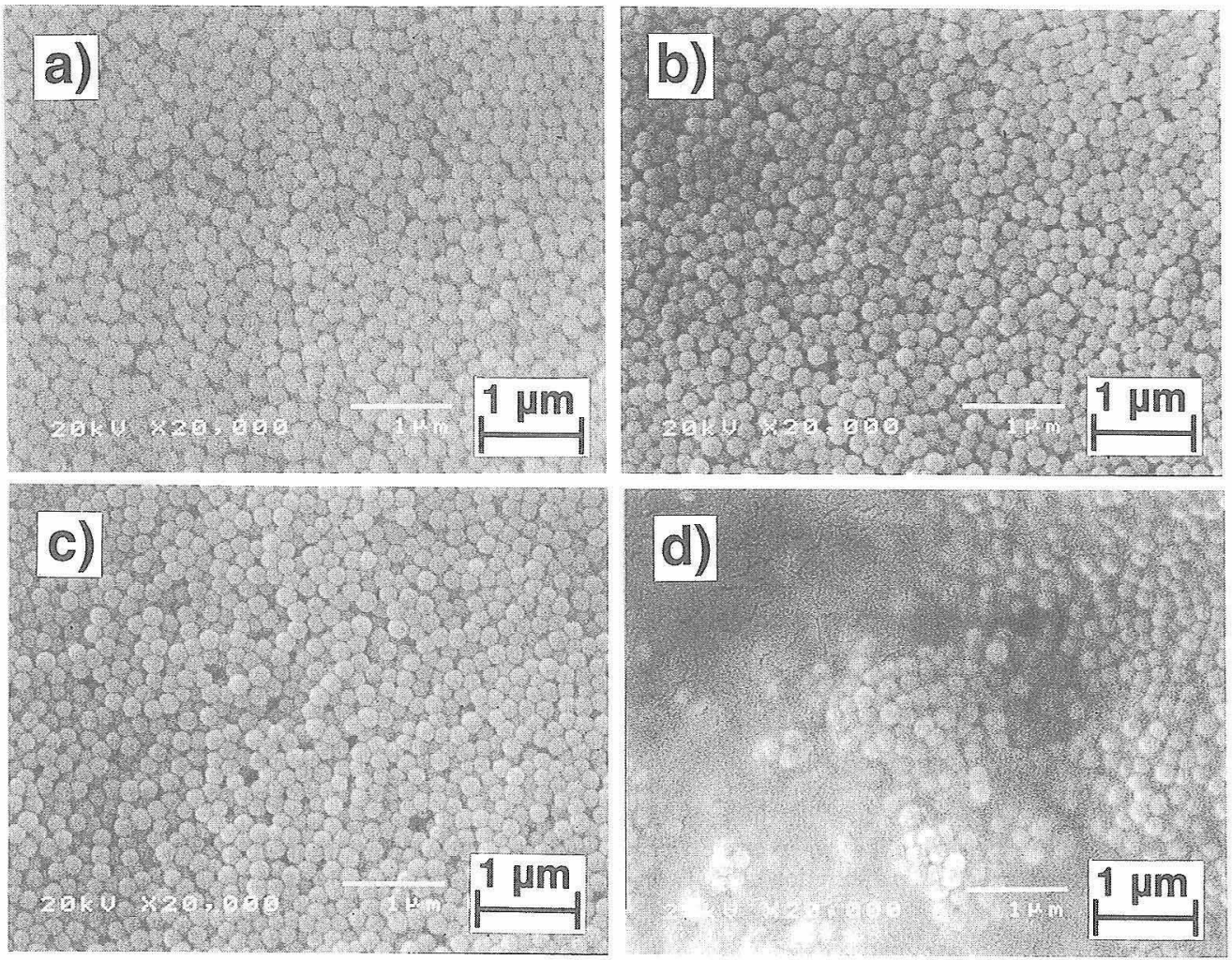

Fig. 6. SEM photographs of the surface of thick silica films. PEI added was (a) 0.01 , (b) 0.10 , (c) 0.50 and (d) 1.00 mass \%

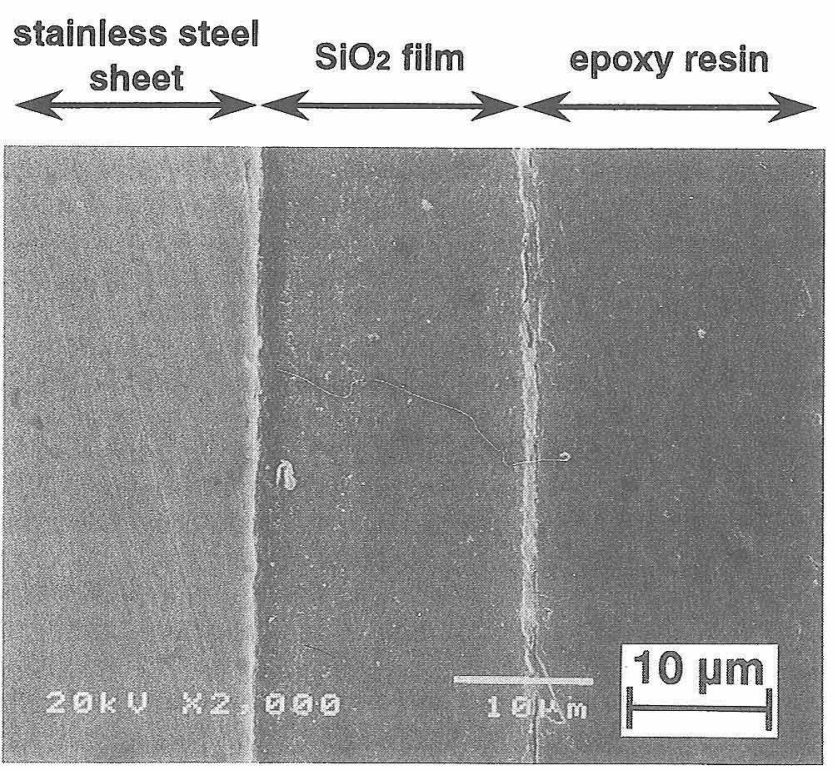

Fig. 7. SEM photograph of the cross section of a thick silica film. Deposition time was 5 min and PEI added was 0.01 mass \%.
0.50 mass \% PEI was caused by the increase of total electricity supplied. However, the weight of the silica particles deposited was decreased again at 1.00 mass\% PEI, which is due to the aggregation and precipitation of silica particles in the coating sols. Particle arrangement on the surface of thick silica films was regular when the addition of PEI was 0.01 mass $\%$. However, particle arrangement became irregular and finally no particle deposited regions near aggregated particles were observed by the addition of more amount of PEI. Thick silica films obtained with ca. $20 \mu \mathrm{m}$ in thickness were well adhered on stainless steel sheet.

\section{References}

1) H. Nishimori, M. Tatsumisago and T. Minami, J. Ceram. Soc. Japan, 103, 78-80 (1995).

2) N. Nikaidoh, Tosoh Gijyutu, 16, No. 11, 61-67 (1977).

3) S. Mikawa, Tosoh Gijyutu, 16, No. 11, 73-77 (1977).

4) G. M. Lindquist and R. A. Stratton, I. Colloid Interface Sci., 55, 45-59 (1976).

5) H. Nishimori, M. Tatsumisago and T. Minami, J. Sol-Gel Sci. Tech., 9, 25-31 (1997).

6) H. Nishimori, M. Tatsumisago and T. Minami, J. Mater. Sci., 31, 6529-34 (1996). 